\title{
Outcome of Pregnancies of Unknown Location: An Observational Study Highlighting the Association with Age and Clinical Features
}

\author{
Shaista Zubair ${ }^{1 *}$, Mary Paul ${ }^{2}$, Nausheen Nabeel ${ }^{3}$, Shantila Momin ${ }^{4}$, Jane Thomas ${ }^{5}$, Noureen \\ Sarwar $^{6}$, Maryam Younus ${ }^{7}$, Neeta Maheshwary ${ }^{8}$, Samreen Farrukh ${ }^{9}$ and Adnan Anwar ${ }^{10}$ \\ ${ }^{1}$ Specialist Obstetrics and Gynecology, Oasis Hospital Abu Dhabi, United Arab Emirates \\ ${ }^{2}$ Specialist, Department of Obstetrics \&Gynecology, RIPAS, Bandar Seri Begawan, Brunei Darussalam \\ ${ }^{3}$ Specialist, Oasis Hospital Al Ain Abu Dhabi, United Arab Emirates \\ ${ }^{4}$ Medical officer, Department O\&G, RIPAS, Bandar Seri Begawan, Brunei Darussalam \\ ${ }^{5} 3 r d$ Year Medical Student, Father Muller Medical College, Mangalore, India \\ ${ }^{6}$ Specialists, O\&G, Oasis Hospital, Al Ain, Abu Shabi, UAE \\ ${ }^{7}$ MSC Statistics, University of Karachi Pakistan \\ ${ }^{8}$ Senior Director Medical and Regulatory and Business Development, Hilton Pharma Pvt Ltd. Pakistan \\ ${ }^{9}$ Medical Officer, JSMU. Pakistan \\ ${ }^{10}$ Senior Lecturer Department of Physiology, Altibri Medical College, Pakistan
}

\begin{abstract}
Received: October 27, 2017; Accepted: November 13, 2017; Published:November 20, 2017
*Corresponding author: Shaista Zubair, MRCOG.UK, MRCPI. Ireland, FCPS, MCPS, Specialist Obstetrics and Gynecology, Oasis Hospital Abu Dhabi, United Arab Emirates, Tel:+971 7401617; E-mail: shaistazubair212@gmail.com
\end{abstract}

\begin{abstract}
Background: Women having pregnancy of unknown location (PUL) can be defined as those having positive pregnancy test when no pregnancy is visualized on transvaginal ultrasound. A pregnancy site is not visualized in 8-31\% of early pregnancy scans, although a lower percentage (8-10\%) has been observed in specialized scanning units.

Objective: To determine the prevalence of pregnancy of unknown location (PUL) and its outcome.

Method: A prospective case series study of pregnant women with suspected early pregnancy complication was carried out. Cases were classified as having a pregnancy of unknown location (PUL) by transvaginal ultrasound. Blood sample was taken on presentation to measure the serum beta human chorionic gonadotropins. Expectant management was done until the pregnancy resulted in predefined outcomes. All collected data were analyzed by employing chi square test using SPSS version 20.

Result: Among 2038 women who were referred for suspected early pregnancy complication, 124(6\%) women with a PUL were recruited into this study. The mean age of the study participants was $29.98 \pm 5.79$ years whereas the mean serum b HCG level was $1446 \pm 1079.63 \mathrm{iu} / \mathrm{l}$. Out of total 2038 patients, $124(6 \%)$ were diagnosed with pregnancy of unknown location (PUL). $13(10.48 \%)$ of them had a previous history of ectopic pregnancy, 32 (26\%) had previous history of miscarriage whereas $16(13 \%)$ had previous history of Caesarean section. After stratifying with respect to symptoms, significant differences were observed between the occurrences of various pregnancy outcomes $(\mathrm{p}=0.001)$.

Conclusion: The study findings revealed that only $6 \%$ of the patients were diagnosed with pregnancy of unknown location (PUL). Furthermore, after stratifying with respect to medical history, duration of disease, symptoms, parity and age, significant differences was observed between the occurrences of various pregnancy outcomes for symptoms only.
\end{abstract}

Keywords: Pregnancy of unknown location; Ectopic Pregnancy; Ultrasonography; Intrauterine Pregnancy and Miscarriage 


\section{Introduction}

The term 'pregnancy of unknown location' (PUL) is used whenever there is no sign of either intra or extra uterine pregnancy or retained products of conception on transvaginal ultrasound, despite a positive pregnancy test. [1] A pregnancy site is not visualised in $8-31 \%$ of early pregnancy scans, although a lower percentage (8-10\%) has been observed in specialised scanning units. [2] The majority of PULs resolve spontaneously, regardless of their location. Therefore, it is important in PUL management to focus on identifying the high risk patients to reduce the number of follow ups and interventions. [3]Expectant management of these patients is imperative to avoid a great number of uterine instrumentation and unnecessary laparoscopies. [4] There are two main concerns related to the management of PUL. Firstly, if the pregnancy is ectopic, being diagnosed late may cause serious effects. As might be expected, ectopic pregnancy is the most feared outcome of the PUL among all possible outcomes. Late diagnosis might increase both mortality and morbidity, decrease success of medical treatment and more frequently requires a surgical intervention, which may further negatively affect fertility in the following years. Secondly, the overtreatment of a PUL might potentially turn into a viable intrauterine pregnancy in the following days. Therefore management of PUL seems to be highly crucial in obstetrics clinics. [5] Ectopic pregnancy remains a leading cause of direct maternal death whose incidence has progressively increased during the past years. [6]

This study was intended to determine the prevalence of pregnancy of unknown location (PUL) and its outcome. Furthermore it was aimed to identify its association with age and clinical features.

\section{Material and Method}

A prospective case series study was performed in the department of gynaecology and obstetrics, RIPAS and Suri Seri Begawan Hospital, Brunei Darussalam from January2014 to December 2014.During this period 2038 patients presented in early pregnancy unit with amenorrhea, vaginal bleeding and pain who had been referred by their GP or the hospital consultant for an ultrasound in the emergency department. A complete medical history was taken and for each woman, age, clinical symptoms (pain and bleeding), menstrual dates, past gynaecological history, endometrial thickness and levels of serum HCG were recorded. This was followed by a thorough physical examination and a transvaginal ultrasound (TVS) in all cases. In women with a positive pregnancy test a PUL was defined, on the basis of transvaginal Scan, if the endometrial cavity was seen empty with no evidence of an intrauterine gestational sac or of any retained product of conception in the absence of visualized extra uterine pregnancy. Women with a positive pregnancy test and suspected early pregnancy complications were examined by transvaginal ultrasound to establish the location and viability of pregnancy. All women with pregnancies that could not be located on the scan had a blood sample taken to quantify the Serum beta human chorionic gonadotropins (beta HCG). Expectant management was done until the pregnancy was identified, the condition resolved spontaneously or an intervention was required because clinical symptoms deteriorated or beta HCG levels did not decline.
The exclusion criteria were visualization of any Intrauterine pregnancy(IUP), Identification of an adnexal mass that was thought to be ectopic, blood in pouch of Douglas on the initial scan, visualization of retained product of conception through the speculum and clinically unstable patient and patients with an acute abdomen. In our study the serum beta HCG levels of 25U/L represented positive pregnancy test. Level of beta HCG was measured again after 48 hours as per study protocol. The follow up consisted of monitoring of the clinical features; measurement of serum beta HCG levels every 48 hours and valuation of TVS findings. These women were followed until the final diagnosis was established as a failing PUL, an intrauterine pregnancy (IUP) or an ectopic pregnancy. Data were collected prospectively only from women classified as having a PUL.

Management of pregnancies of unknown location led to four possible outcomes i.e. spontaneous resolution, ectopic pregnancy, IUP and persisting PUL. Normal pregnancy was diagnosed when gestational sac was visualized on TVS within endometrial cavity. These women were re-scanned 7-10 days later to confirm viability. Miscarriage was diagnosed either histological following surgical evacuation of the uterine content or on ultrasound examination in pregnancies that progressed to develop a demonstrable intrauterine gestational sac that subsequently failed in the first trimester. Ectopic Pregnancies were diagnosed on follow up when TVS revealed a heterogeneous mass seen in the adnexal region adjacent to the ovary, a mass with a hyper echogenic ring around the gestational sac or the presence of embryo with or without a heartbeat in the adnexal accompanied by raised serum level of beta human chorionic gonadotropins. Spontaneous resolution was defined by a reduction in serum b HCG levels to $<25 \mathrm{iu} / \mathrm{l}$ with a complete resolution of symptoms without the need for any therapeutic intervention so the location of pregnancies therefore remained unknown.

Data were cleaned and then analyzed through the SPSS version 20.Meansand standard deviations were calculated for continuous variables such as age, duration of disease and beta HCG level. Frequencies and percentages were calculated for categorical variables like the outcome of the PUL and medical history of ectopic pregnancy, miscarriage, Caesarean Section, symptoms and parity. Stratification with respect to age, parity, duration of disease and symptoms was done. Post stratification Chi-square test was applied for univariate analysis of all the parameters. $\mathrm{P}<0.05$ was taken as significant.

\section{Results}

The study results revealed that the mean age of the study participants was29.98 \pm 5.79 years whereas the mean serum b HCG level was $1446 \pm 1079.63 \mathrm{iu} / \mathrm{l}$. Out of total 2038 patients, $124(6 \%)$ were diagnosed with pregnancy of unknown location (PUL). $13(10.48 \%)$ of them had a previous history of ectopic pregnancy,32 (26\%) had previous history of miscarriage whereas $16(13 \%)$ had previous history of Caesarean section. 63 (51\%) of them presented with vaginal bleeding at the initial visit with or without abdominal pain, 31 (25\%) presented with lower abdominal pain and only 5 (4\%) women presented with triad of amenorrhea, pain and bleeding. 
At follow up the observed outcome of these124 patients revealed that $44(35 \%)$ cases had spontaneous resolution, 40 cases (32\%) were diagnosed to have ectopic pregnancy, 22 (18\%) cases were diagnosed with normal intrauterine true pregnancies with positive pregnancy test, 13 cases $(10 \%)$ had miscarriage of an IUP and 5 cases (4\%) remained as cases of persisting PUL. Surgical interventions were required only in $20(16.1 \%)$ cases; $2(1.61 \%)$ uterine curettages in the miscarriage group, $12(9.6 \%)$ laparoscopy and $6(4.8 \%)$ laparotomies for treatment of ectopic pregnancies, whereas $10(8.1 \%)$ patients received MXT. The mean follow up period was 6 weeks (ranged from 4 to 14 weeks).

After stratifying with respect to medical history, duration of disease, parity and age, no significant differences was observed between the occurrences of various pregnancy outcomes whereas after stratifying with respect to symptoms, significant differences were observed between the occurrences of various pregnancy outcomes ( $\mathrm{p}=0.001)$ (Figure 1) (Table 1).

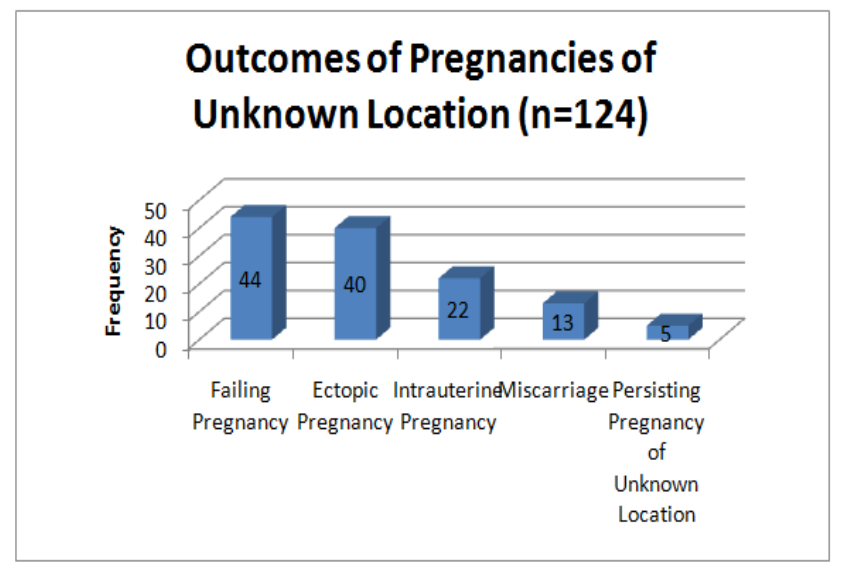

Figure 1

\begin{tabular}{|c|c|c|c|c|c|c|c|}
\hline Variables & $\begin{array}{l}\text { Failing } \\
\text { Pregnancy } \\
(n=44)\end{array}$ & $\begin{array}{l}\text { Ectopic } \\
\text { Pregnancy } \\
(n=40)\end{array}$ & $\begin{array}{c}\text { Intrauterine } \\
\text { Pregnancy } \\
(n=22)\end{array}$ & Miscarriage(n=13) & $\begin{array}{c}\text { Persisting } \\
\text { Pregnancy } \\
\text { of Unknown } \\
\text { Location }((n=05))\end{array}$ & Overall((n=124)) & P-values \\
\hline Age & $30.62 \pm 6.04$ & $30 \pm 5.63$ & $27.90 \pm 5.43$ & $32.18 \pm 6.91$ & $30.5 \pm 3.01$ & $29.98 \pm 5.79$ & -------- \\
\hline b HCG level & $869.18 \pm 105.74$ & $1925.08 \pm 1184.04$ & $1399.1 \pm 1115.28$ & $1596.14 \pm 1293.98$ & $1468 \pm 988$ & $1446 \pm 1079.63$ & -------- \\
\hline \multicolumn{8}{|c|}{ Medical history } \\
\hline Ectopic & $5(11.36 \%)$ & $8(20 \%)$ & - & - & - & $13(10.48 \%)$ & \multirow{3}{*}{0.125} \\
\hline Miscarriage & $9(20.45 \%))$ & $10(25 \%)$ & $4(18.18 \%)$ & $6(46.15 \%)$ & $3(60 \%)$ & $32(25.80 \%)$ & \\
\hline $\begin{array}{c}\text { Caesarean } \\
\text { section }\end{array}$ & $5(11.36 \%)$ & $8(20 \%)$ & $1(4.54 \%)$ & $2(15.38 \%)$ & - & $16(12.90 \%)$ & \\
\hline \multicolumn{8}{|c|}{ Duration of disease } \\
\hline$\leq 6$ weeks & 25 & 24 & 18 & 3 & 4 & $6.29 \pm 2.24$ & \multirow{3}{*}{0.069} \\
\hline 7-8 weeks & 14 & 12 & 2 & 6 & 1 & $(4-12)$ & \\
\hline$>8$ weeks & 5 & 4 & 2 & 4 & 0 & & \\
\hline \multicolumn{8}{|l|}{ Symptoms } \\
\hline GA & 10 & 8 & 5 & - & 2 & $25(20.16 \%)$ & \multirow{4}{*}{0.001} \\
\hline Bleed & 29 & 17 & 5 & 10 & 2 & $63(50.80 \%)$ & \\
\hline LAP & 5 & 10 & 12 & 3 & 1 & $31(25 \%)$ & \\
\hline Triad & - & 5 & - & - & - & $5(4 \%)$ & \\
\hline \multicolumn{8}{|l|}{ Parity } \\
\hline Nullipara & 12 & 15 & 12 & 4 & 4 & $47(37.90 \%)$ & \multirow{3}{*}{0.068} \\
\hline Multipara & 18 & 18 & 9 & 4 & 1 & $50(40.32 \%)$ & \\
\hline Grandpara & 14 & 7 & 1 & 5 & 0 & $27(21.77 \%)$ & \\
\hline
\end{tabular}

Citation: Shaista Z, Mary P, Nausheen N, et al. (2017) Outcome of Pregnancies of Unknown Location: An Observational Study Highlighting the Association with Age and Clinical Features. SOJ Gynecol Obstet Womens Health 3(3):1-4. DOI: http://dx.doi. 


\begin{tabular}{|c|c|c|c|c|c|c|}
\hline $\begin{array}{c}\text { Less than } \\
26 \text { years }\end{array}$ & 11 & 12 & 9 & 3 & - & $35(28.23 \%)$ \\
\hline $\begin{array}{c}26-35 \\
\text { years }\end{array}$ & 24 & 19 & 12 & 7 & 5 & $67(54.03 \%)$ \\
\hline $\begin{array}{c}\text { Above } 35 \\
\text { years }\end{array}$ & 9 & 9 & 1 & 3 & - & 0.324 \\
\hline
\end{tabular}

\section{Discussion}

Whenever a women presents with a positive pregnancy test but no evidence of pregnancy on transvaginal ultrasound, clinical assessment and serum b HCG estimation should be carried out [7] TVS is an accurate diagnostic procedure for women with suspected early pregnancy complications. It enables diagnosis of location of these pregnancies in greater than $90 \%$ of cases. [8] In this study accurate location of the pregnancy was determined at the initial visit in $94 \%$ of cases whereas the remaining $6 \%$ were cases of PUL. The international society of ultrasound in obstetrics and Gynecology states that early pregnancy units should try to maintain a PUL rate at less than 15\%. [9] The concept of combining ultrasound with serum b HCG using a discriminatory zone has been widely evaluated and the current recommended value ranges from 1000-2400 iu/l. [10-12] Expectant management of PUL has been shown to be safe for the majority of asymptomatic hemodynamically stable women.[13,14] According to the Association of Early pregnancy units guidelines, if no intrauterine or ectopic pregnancy or retained products of conception are seen on transvaginal ultrasound and the woman is asymptomatic at initial assessment, she can be managed conservatively.[12] This is irrespective of the b HCG discriminatory zone and additional ultrasound findings, such as a suspicious adnexal mass $<3 \mathrm{~cm}$. Conservative management involves estimation of serum $b$ HCG levels at 48 hours to determine the pattern of b HCG change from the initial assessment. Further follow-up with b HCG and transvaginal ultrasound can be arranged or therapeutic intervention made. [12] The pattern of rise or fall in b HCG after 48 hours and the b HCG ratio are useful in distinguishing between puls developing into failing puls from intrauterine and ectopic pregnancy.[2]It predicts an intrauterine pregnancy with a positive predictive value of $96.5 \%$.[15]

Literature suggests that only some cases require diagnostic laparoscopy or uterine curettage to determine the location of pregnancy but there is no consensus on what is an acceptable intervention rate in this group. In this study, only $20(16.1 \%)$ women required surgical intervention; $12(9.6 \%)$ laparoscopies were performed and $6(4.8 \%)$ laparotomies to treat ectopic pregnancies while only $2(1.61 \%)$ cases required uterine evacuation in the miscarriage group. However, an expectant management is appropriate for patients who are hemodynamically stable.

\section{Conclusion}

The study findings revealed that only $6 \%$ of the patients were diagnosed with pregnancy of unknown location (PUL) Furthermore, after stratifying with respect to medical history, duration of disease, symptoms, parity and age, significant differences was observed between the occurrences of various pregnancy outcomes for symptoms only.

\section{References}

1. Royal College of Obstetricians and Gynaecologists. The Management of Early Pregnancy Loss. Green- top Guideline No. 25. London; RCOG: 2006.

2. Haritha Sagili, kamel Mohamed. Pregnancy of unknown location: an evidence -based approach to management. Journal the TOG. 2008;10:224-230. Doi: 10.1576/toag.10.4.224.27438

3. CordinaM, Schram-Gajraj K, Ross JA, Lautman K, Jurkovic D. Introduction of a single visit protocol in the management of selected patients with pregnancy of unknown location: a prospective study. BJOG. 2011;118(6):693-697. Doi:10.1111/j.14710528.2011.02893.x

4. Monia Malek-mellouli, Maïna Oumara, Fethi Ben Amara, Ons Zouch, Khaled Neji, Hédi Reziga. prediction of ectopic pregnancy in early pregnancy of unknown location. La tunisie Medicale. 2013;91(1):27-32.

5. Gokhan Boyraz and GurkanBozdaq. Pregnancy of unknown location. J Turk Gynecol Assoc. 2013;14(2):104-108. Doi: 10.5152/ jtgga.2013.74317

6. Condous G, Okaro E, Bourne T. The conservative management of early pregnancy complications: a review of the literature. Ultrasound Obstet Gynecol. 2003;22:929-934. Doi: 10.1002/uog.236

7. condous G, ThomasJ, OkaroE, Bourne T. Placenta site trophoblastic tumour masquerading as an ovarian ectopic pregnancy. Ultrasound Obstet Gynecol. 2003;21(5):504-506. Doi:10.1002/uog.106

8. Moschos E, twickler M. Endometrial thickness predicts intrauterine pregnancy in patient with pregnancy of unknown location. Ultrasound obstetrics Gynecol. 2008;32(7):929-934. Doi: 10.1002/ uog. 6248

9. Condous G, Kirk E, Van calster B, Van Huffel S, Timmerman D, Bourne T. Failing pregnancies of unknown location; a prospective evaluation of the humen chorionic gonadotrophin ratio. $\mathrm{Br} J$ Obstet Gynecol. 2006;113(5):521-527. Doi: 10.1111/j.1471-0528.2006.00924.x

10. Cacciatore B, Stenman UH, Ylötsalo P. Diagnosis of ectopic pregnancy by vaginal ultra sonography in combination with a discriminatory serum hCG level of 1000 IU/L (IRP). BJOG. 1990;97(10):904-908. Doi:10.1111/j.1471-0528.1990.tb02445.x

11. Ankum WM, HajeniusPJ, Schrevel LS, Vander Veen F. Management of suspected ectopic pregnancy. Impact of new diagnostic tools in 686 consecutive cases. JReprod Med. 1996;41(10):724-728.

12. Association of Early Pregnancy Units. Guidelines. 2007.

13. Hahlin M, Thorburn J, Bryman I. The expectant management of early pregnancies of uncertain site. Hum Reprod. 1995;10(5):1223-1227.

14. Banerjee S, Aslam N, Woelfer B, Lawrence A, Elson J, Jurkovic D. Expectant management of early pregnancies of unknown location: a prospective evaluation of methods to predict spontaneous resolution of pregnancy. Br j Obstet gynecol. 2001;108(2):158-163.

15. Condous G, Okaro E, Khalid A, Zhou Y, Lu C, Van Huffel S, et al. Role of Biochemical and ultrasonographic indices in the management of pregnancies of unknown location. Ultrasound Obstet Gynecol. 2002;20(Suppl 1):36-37. 